\title{
Editorial
}

\section{Inequalities in the A-Harmonic Equations and the Related Topics}

\author{
Shusen Ding, ${ }^{1}$ Yong Wang, ${ }^{2}$ and Yuming Xing ${ }^{2}$ \\ ${ }^{1}$ Department of Mathematics, Seattle University, Seattle, WA 98122, USA \\ ${ }^{2}$ Department of Mathematics, Harbin Institute of Technology, Harbin, 150001, China \\ Correspondence should be addressed to Shusen Ding, sding@seattleu.edu
}

Received 24 November 2010; Accepted 24 November 2010

Copyright (c) 2010 Shusen Ding et al. This is an open access article distributed under the Creative Commons Attribution License, which permits unrestricted use, distribution, and reproduction in any medium, provided the original work is properly cited.

In order to stimulate further investigation on inequalities involved in the $A$-harmonic equation and the related topics, to provide readers with a rich research resource, and to build up a timely communication stage for our colleagues, we determined to edit and publish this special issue. The $A$-harmonic equations belong to the nonlinear elliptic equations written in terms of an operator $A$, which are of particular importance because they have wide applications in many fields, including quasiconformal analysis, nonlinear elasticity, and potential theory. The $A$-harmonic equations are extensions of the usual $p$-harmonic equation. The developments of the theory about the $A$-harmonic equation are closely related to the theory of quasiconformal and quasiregular mappings. A series of results about the solutions to different versions of the $A$-harmonic equations and applications have been developed during the last 40 years.

During the open period of this special issue, more than 50 manuscripts were submitted to this special issue for consideration of publication. After a critical review process, 32 strong papers among these submissions had been accepted for publication in this special issue. We are very pleased to see that various versions of inequalities involved in the $A$ harmonic equation and the related topics have been developed and studied among these 32 accepted papers. Specifically, 16 papers are in the area of the $A$-harmonic equations and the estimates for solutions of some versions of equations, such as "Some estimates of integrals with a composition operator" by B. Liu, "A-harmonic equations and the Dirac operator" by C. A. Nolder, "Global estimates for singular integrals of the composition of the maximal operator and the Green's operator" by Y. Ling and H. M. Umoh and "Some priori estimates about solutions to nonhomogeneous A-harmonic equations" by J. Zhu and J. Li. Two papers ("Wiman and Arima theorems for quasiregular mappings" by $\mathrm{O}$. Martio and "On harmonic quasiconformal quasiisometries" by M. Mateljević and M. Vuorinen) belong to quasiconformal analysis. Three 
papers deal with solution properties of the diffusion equation and the nonlinear parabolic problems. Two papers are in the areas of special functions and the Orlicz sequence space. One paper is in matrix analysis. Eight papers are about the operator theory and functional equations. Operators are critical tools to establish existence and regularity for solutions to various partial differential equations, including the $A$-harmonic equations, and to control oscillatory behavior in different domains. In many situations, the process to solve a partial differential equation or a system of the partial differential equations involves integration or integral estimate (for numerical solutions), and the operators are often used to represent solutions. Hence, the investigation on operators and related operator equations are very important to many fields of mathematics.

We strongly believe that the results established in these 32 papers will develop broad and rigorous conceptual understanding of the $A$-harmonic equations and the related topics and will explore the further applications in different fields of mathematics.

Shusen Ding

Yong Wang

Yuming Xing 